\title{
ABLAÇÃO QUÍMICA DO CORPO CILIAR EM GLAUCOMA SECUNDÁRIO EM CÃO: RELATO DE CASO
}

\author{
Thaís Lucielle Vitor ${ }^{1}$; Taiza Gonçalves de Freitas ${ }^{1}$; Larissa Vieira de Paula ${ }^{1}$; Leandro \\ Rodrigues de Oliveira Carvalho"; Andréia Vitor Couto do Amaral² \\ 1 Residente do Hospital Veterinário da Universidade Federal de Jataí (UFJ) \\ (thais_lucielle@hotmail.com) \\ 2 Docente do curso de Medicina Veterinária da Universidade Federal de Jataí (UFJ) \\ Recebido em: 22/09/2018 - Aprovado em: 23/11/2018 - Publicado em: 03/12/2018 \\ DOI: 10.18677/EnciBio_2018B3
}

\begin{abstract}
RESUMO
Glaucoma é uma enfermidade oftálmica não infecciosa, multifatorial, que acomete seres humanos e animais domésticos. Ocasiona degeneração progressiva do nervo óptico e morte de células ganglionares, podendo levar a perda da visão de forma irreversível. Ocorre aumento da pressão intraocular (PIO), por diminuição da drenagem do humor aquoso ( $\mathrm{HO})$, principalmente pelo ângulo iridocorneal. $\mathrm{O}$ glaucoma é classificado em primário (aberto, estreito ou fechado), secundário, congênito e também como agudo, crônico ou absoluto. Os sinais clínicos incluem vasos episclerais engurgitados, midríase, edema de córnea, dor, buftalmia, lagoftalmia, fraturas da membrana de Descemet e cegueira. O diagnóstico é baseado nas manifestações clínicas associado com a utilização de tonometria, gonioscopia, ultrassonografia e eletrorretinografia. $O$ tratamento é feito com base em fármacos que diminuem a PIO, podendo ser necessária associação com procedimentos cirúrgicos. As técnicas cirúrgicas objetivam diminuir a produção do $\mathrm{HO}$, lesionando o corpo ciliar ou incrementando sua drenagem, através de vias alternativas. Em estágios finais do glaucoma pode-se optar pela realização de enucleação. Este artigo relata um caso atendido no Hospital Veterinário da UFJ (Universidade Federal de Jataí), de uma cadela, raça Shih tzu, três anos de idade, que apresentou buftalmia acentuada, alterações da superfície corneal e da câmara anterior do olho esquerdo. Por meio da anamnese, exame físico e exames auxiliares, chegou-se ao diagnóstico de glaucoma secundário com perda da visão. 0 tratamento realizado foi a ablação química do corpo ciliar, utilizando injeção intravítrea de gentamicina e dexametasona, obtendo-se a diminuição do bulbo ocular, diminuição da opacidade corneal e redução da PIO.
\end{abstract}

PALAVRAS-CHAVE: cegueira, gentamicina, glaucoma absoluto

\section{CHEMICAL ABLITION OF THE CILIAR BODY IN SECONDARY GLAUCOMA IN DOG: CASE REPORT}

\section{ABSTRACT}

Glaucoma is a non-infectious, multifactorial ophthalmic disease that affects humans and domestic animals. It causes progressive degeneration of the optic nerve and death of ganglion cells, which can cause irreversible loss of vision. It occurs mainly ENCICLOPÉDIA BIOSFERA, Centro Científico Conhecer - Goiânia, v.15 n.28; p. 22 2018 
due to an increase in intraocular pressure due to decreased drainage of the aqueous humor $(\mathrm{HO})$, mainly due to the iridocorneal angle. Glaucoma is classified into primary (open, narrow or closed), secondary, congenital and also as acute, chronic or absolute. Clinical signs include episclerial vessels engurged, mydriasis, corneal edema, pain, bufftalia, lagophthalmia, Descemet's membrane fractures and blindness. The diagnosis is based on the clinical manifestations associated with the use of tonometry, gonioscopy, ultrasonography and electroretinography. The treatment is based on drugs that decrease IOP, and it may be necessary to be

associated with surgical procedures. Surgical techniques aim to reduce $\mathrm{HO}$ production, injuring the ciliary body or increasing its drainage, through alternative routes. In the final stages of glaucoma, enucleation can be performed. This article reports a case attended at the Veterinary Hospital of the UFJ (Federal University of Jataí) of a three-year-old Shihtzu breed, which presented left eye volume increase and corneal surface alterations, and the findings in the anamnesis and physical examination suggested the diagnosis of glaucoma. The treatment was the chemical ablation of the ciliary body using intravitreal injection of gentamicin and dexamethasone, with the subsequent result of ocular bulb reduction, reduction of corneal opacity and reduction of IOP.

KEYWORDS: blindness, gentamicin, secondary glaucoma

\section{INTRODUÇÃO}

O glaucoma é uma importante afecção oftálmica, tanto na medicina veterinária quanto na humana. Trata-se de uma condição dolorosa e que se não for diagnosticada ou tratada precocemente pode causar perda irreversível da visão, além de ser considerado a principal causa de enucleação de olhos em cães. (OFRI; NARSFTROM, 2007; STROM et al., 2011; SANDALON et al., 2013; KATO; WOERDT, 2017) Nos animais domésticos é mais prevalente na população canina, podendo acometer cerca de $0,5 \%$ dos cães, mas em algumas raças pode chegar a $5 \%$ (MARTINS et al., 2009).

É definido como uma neuropatia óptica progressiva multifatorial, possuindo o aumento da PIO como sua principal característica, sem, entretanto, ser um achado patognomônico (MILLER; BENTLEY, 2015; KATO; WOERDT, 2017). O aumento da PIO é ocasionado pela redução ou obstrução da drenagem do humor aquoso (ORIÁ, 2013).

Apesar do aumento da PIO estar presente na maioria dos casos de glaucoma, sabe-se que os danos ao nervo óptico e tecidos adjacentes podem ocorrer antes que a elevação da mesma ocorra ou mesmo com seu controle. (MILLER; BENTLEY, 2015).

Suspeita-se de glaucoma quando a PIO se demonstrar aumentada durante o exame clínico. A PIO normal de cães varia entre 15 e $30 \mathrm{mmHg}$ (BROADWATER et al., 2008). A PIO representa o equilíbrio entre a produção de humor aquoso e o fluxo de saída. Existem muitos mecanismos fisiopatológicos que podem ameaçar esse equilíbrio ao diminuir a drenagem do humor aquoso, levando ao glaucoma e causando danos intraoculares. Entretanto, não existe evidência de algum mecanismo fisiopatológico que aumente a produção do aquoso. (BRAS; MAGGIO, 2015).

O humor aquoso $(\mathrm{HO})$ tem como principais papéis o fornecimento de nutrição, remoção de resíduos metabólicos, e manutenção da $\mathrm{PIO}$ em níveis normais (PIZZIRANI; GONG, 2015). O HO tem sua produção na câmara posterior do olho ENCICLOPÉDIA BIOSFERA, Centro Científico Conhecer - Goiânia, v.15 n.28; p. $23 \quad 2018$ 
pelo corpo ciliar, e através da fenda pupilar passa para a câmara anterior, preenchendo-a (ORIÁ et al., 2013).

As vias de drenagem do humor aquoso constituem principalmente em duas, a convencional e a não convencional. A via convencional é responsável pela drenagem de 85 a 90\% na espécie canina e é chamado ângulo de drenagem iridocorneal, a via não convencional é responsável por pequena parte da drenagem e é conhecido como ângulo de drenagem uveoescleral (EURIDES et al., 2013, ORIÁ et al., 2013).

Alguns autores atribuíram mais mecanismos, além da elevação da $\mathrm{PIO}$, como possíveis causadores de glaucoma, tais como defeitos na microcirculação e redução do fluxo axoplasmático do nervo óptico, liberação de glutamato pelos axônios lesionados e influência de moléculas neuroexcitatórias (WERNE et al., 2008; CAPRIOLI; COLEMAN, 2010). Outros fatores de risco são considerados importantes na ocorrência desta oftalmopatia, como idade, histórico familiar, raças específicas e gênero (PIZZIRANI, 2015).

O glaucoma nos animais pode ser classificado com relação a sua possível causa como primário, secundário ou congênito, com relação à aparência do ângulo de drenagem como glaucoma de ângulo aberto, de ângulo estreito ou de ângulo fechado, ou quanto a duração ou estágio da doença, como agudo, crônico ou absoluto. Estas classificações podem ser usadas em combinação para oferecer uma descrição mais detalhada de um paciente individual. A classificação tem ramificações importantes para o tratamento e prognóstico. (PIZZIRANI, 2015; MILLER; BENTLEY, 2015).

O glaucoma congênito é uma forma grave de glaucoma que se desenvolve imediatamente após o nascimento ou nos primeiros meses de vida. O termo congênito pode sugerir que a condição está presente no nascimento, entretanto as anormalidades do ângulo podem estar presentes no nascimento e o aumento da PIO e consequentes manifestações clínicas da doença podem ocorrer mais tarde (PIZZIRANI, 2015).

O glaucoma é classificado como primário quando ocorre devido à obstrução da drenagem do humor aquoso pelo ângulo de drenagem, sem nenhuma doença ocular preexistente. Ambos os olhos são acometidos, entretanto a manifestação clínica da doença pode ocorrer em momentos diferentes entre um olho e outro no mesmo paciente (PLUMMER, 2013). Esse tipo de glaucoma possui caráter hereditário em algumas raças caninas, sendo relatada a presença em aproximadamente 45 raças, dentre as mais propensas estão o Cocker Spaniel Americano, o Beagle, o Basset Hound, o Samoyeda, o Husky Siberiano, o Shar Pei, o Poodle e o Elkhound Noruegues (BROOKS, 2008).

O glaucoma secundário é mais comum em cães em comparação com o primário e ocorre quando outra afecção intraocular resulta em comprometimento do fluxo do humor aquoso (OLIVER, 2015). As causas primárias mais comumente associadas a esta condição são luxações ou subluxações da lente, cataratas, uveítes, úlceras de córnea, neoplasias intraoculares, traumas ou complicações pósoperatórias (MARTINS et al., 2009).

De acordo com a duração, o glaucoma crônico se manifesta com a elevação progressiva da $\mathrm{PIO}$, em contrapartida, o agudo caracteriza-se por sua elevação súbita (BROOKS, 2008). Casos agudos representam emergências oftálmicas, devendo ser imediatamente abordados com o objetivo de preservação a visão (KATO; WOERDT, 2017). 
O tipo absoluto é o estágio final do glaucoma, resultante da PIO elevada de forma persistente e irresponsiva aos tratamentos. Nestes casos, observa-se perda de visão por atrofia progressiva da cabeça do nervo óptico, com presença de buftalmia, alterações degenerativas graves na maioria dos tecidos oculares, perda da visão e, com frequência, dor (SLATTER, 2005).

Os sinais clínicos variam dependendo da causa, do estágio da doença, do tipo do glaucoma e da extensão e duração da elevação da PIO, os comumente observados são: hiperemia conjuntival, vasos episclerais engurgitados, midríase, lacrimejamento, edema e úlceras corneanas, luxação ou subluxação da lente, buftalmia, fraturas da membrana de descemet, dor e cegueira (MILLER; BENTLEY, 2015).

Para o diagnóstico de glaucoma é importante realizar a identificação correta dos sinais clínicos característicos, juntamente com um exame físico completo e realização de métodos diagnósticos, como a tonometria, a gonioscopia e a fundoscopia. Existem também outros métodos, que quando disponíveis, são de grande valia no diagnóstico do glaucoma, como a ultrassonografia e a eletroretinografia (MILLER; BENTLEY, 2015; PUMPHREY, 2015).

A tonometria é o método mais comumente utilizado quando há suspeita de glaucoma, e consiste na técnica de avaliação da PIO, pois quando a PIO está muito elevada suspeita-se da doença (MARTINS et al., 2009). Porém em glaucoma secundário a uveíte deve-se ter atenção, pois geralmente nesses pacientes o valor da PIO é baixo, devido à inflamação e ao edema do corpo ciliar, mediados por prostaglandinas que impedem a formação do humor aquoso (VEIGA et al. 2013).

O tratamento do glaucoma pode ser clínico ou cirúrgico, e muitas vezes pode necessitar da associação de ambos (TURNER, 2010). O tratamento do glaucoma tem como objetivos a prevenção da lesão ao nervo óptico, preservação da função visual e diminuição da dor. A terapia antiglaucomatosa é direcionada basicamente à redução da PIO. O tratamento cirúrgico do glaucoma é direcionado para alterar a produção de humor aquoso, a drenagem ou a combinação de ambos (SEBBAG et al., 2018).

O tratamento para o glaucoma secundário consiste em tratar, sempre que possível, a doença oftálmica primária, juntamente com a terapia antiglaucomatosa visando à redução da PIO. O tratamento médico do glaucoma objetiva controlar o aumento da PIO pela aplicação tópica de uma variedade de medicamentos, e quando necessário, realização de procedimentos cirúrgicos. Diferentes categorias de medicamentos antiglaucomatosos podem ser usados em combinação devido à sua contribuição complementar à redução da PIO (KATO; WOERDT, 2017).

Os fármacos utilizados no manejo clínico do glaucoma são classificados conforme seu mecanismo de ação. Existem os fármacos que aumentam a drenagem do humor aquoso sem reduzir sua formação (agentes colinérgicos e análogos das prostaglandinas), agentes que reduzem a formação do humor aquoso (agonistas adrenérgicos e inibidores da anidrase carbônica) e agentes que cursam com ambos mecanimos de ação (antagonistas adrenérgicos). Os diuréticos osmóticos (exemplo, manitol), podem ser utilizados em casos agudos, quando a PIO está excessivamente elevada (MARTINS et al., 2009).

Os agentes inibidores da anidrase carbônica constituem fármacos amplamente utilizados para o tratamento do glaucoma, indicados para praticamente todos os tipos da doença (BORGES, 2007). Comercialmente, está disponível a 
dorzolamida a $2 \%$ associada com ao maleato de timolol a 0,5\% (PLUMER et al., 2006).

O glaucoma pode se tornar refratário à terapia medicamentosa no percurso do tratamento, sendo necessária intervenção cirúrgica para alivio da dor e desconforto, principalmente em casos secundários a afecções inflamatórias. Nesses casos opta-se geralmente por técnicas que ocasionem redução da produção do humor aquoso, por lesarem o corpo ciliar. As mais comumente utilizadas são as que ocasionam destruição parcial do corpo ciliar (ciclodiatermia e ciclocrioterapia), ou destruição total (com utilização de produtos farmacológicos) (ORIÁ, 2013; PUMPHREY, 2015).

Nos casos de cegueira irreversível, a administração intravítrea de gentamicina e dexametasona tem sido utilizada para promover necrose do corpo ciliar, com o objetivo de causar redução da produção do humor aquoso e analgesia, sob condições estéticas aceitáveis. Os resultados são variáveis, podendo ocasionar desde opacidade e redução do tamanho do olho, hemorragia ocular, inflamação persistente e catarata (EURIDES, 2013).

A técnica de ablação por gentamicina é realizada aproximadamente 6 a $8 \mathrm{~mm}$ após o limbo, onde se insere uma agulha hipodérmica calibre 20 a uma profundidade de $1 \mathrm{~cm}$ em direção ao nervo óptico. Então aspira-se 0,5 a $1 \mathrm{ml}$ do vítreo movimentando a agulha várias vezes dentro do corpo vítreo para auxiliar a aspiração, em seguida é acoplada outra seringa e injetado $25 \mathrm{mg}$ de gentamicina e 1 mg de dexametasona (EURIDES, 2013).

$\mathrm{Na}$ técnica de ablação química do corpo ciliar é realizada uma destruição farmacológica por necrose tóxica do corpo ciliar mediante a injeção intravítrea de sulfato de gentamicina e dexametasona (BROOKS, 2008; MARTINS et al., 2009). Esta técnica pode ser empregada somente em casos em que não haja presença de neoplasia e quando já houver controlado inflamações oculares (PUMPHREY, 2015).

Quando o glaucoma encontra-se em estágio final da PIO crônica, com cegueira, buftalmia, alteração degenerativa e dor, pode-se optar pela realização da enucleação, podendo também ser realizada a evisceração, ou evisceração com colocação de prótese, dependendo do caso (TURNER, 2010).

O objetivo deste trabalho é relatar um caso de ablação química do corpo ciliar com gentamicina e dexametasona no olho de um cão com diagnóstico de glaucoma absoluto, inicialmente secundário, não responsivo ao tratamento clínico.

\section{RELATO DE CASO}

Foi atendida, no Serviço de Oftalmologia Veterinária do Hospital Veterinário da Regional Jataí, UFG, uma cadela, raça Shih tzu, três anos de idade, com as queixas principais de aumento de volume do olho esquerdo e alterações da superfície corneal. A tutora relatou que a paciente havia sofrido um trauma ocular provocado por um gato, há cerca de 60 dias, sendo tratada com colírios a base de tobramicina, tobramicina associada com dexametasona, prednisolona e atropina. Entretanto, não apresentou melhora do quadro, com aumento do volume do globo ocular, resolvendo assim, buscar um especialista em oftalmologia.

Ao exame oftálmico, foram verificados no olho esquerdo buftalmia, vasos episclerais ingurgitados, ausência de resposta à ameaça e epífora. À biomicroscopia utilizando-se lâmpada de fenda (SL-15 Kowa) foram visibilizados edema de córnea acentuado, estrias na membrana de descemet, neovascularização e pigmentação corneal de forma moderada e secreção seromucosa de coloração amarelo 
esverdeada. A PIO, aferida após a instilação de uma gota de colírio anestésico a base de proximetacaína a $0,5 \%$, foi de $46 \mathrm{mmHg}$ (Tono-pen XL). O exame da câmara anterior e a fundoscopia foi comprometido em decorrência da intensa opacidade da córnea. No olho direito não foram encontradas alterações, e a PIO foi $22 \mathrm{mmHg}$. A prova pelo tingimento com fluoresceína foi negativa em ambos os olhos (Fluorescein strips, Ophthalmos, SP).

Diante do quadro, foi prescrito colírio à base de dorzolamida associado com timolol (Cosopt $\AA$ ) três vezes ao dia, colírio a base de moxifloxacino (Vigamox®) seis vezes ao dia e gel a base de carbômer (Vidisic $®$ ) de quatro a seis vezes ao dia, por dez dias.

Decorridos dez dias, durante o retorno, observou-se piora do quadro. A paciente apresentava aumento da buftalmia, com intensa hiperemia e ingurgitamento dos vasos episclerais, apesar do tratamento instituído (Figura 1). A PIO continuava em $46 \mathrm{mmHg}$. Foi então indicada a ablação química do corpo ciliar utilizando injeção intravítrea.

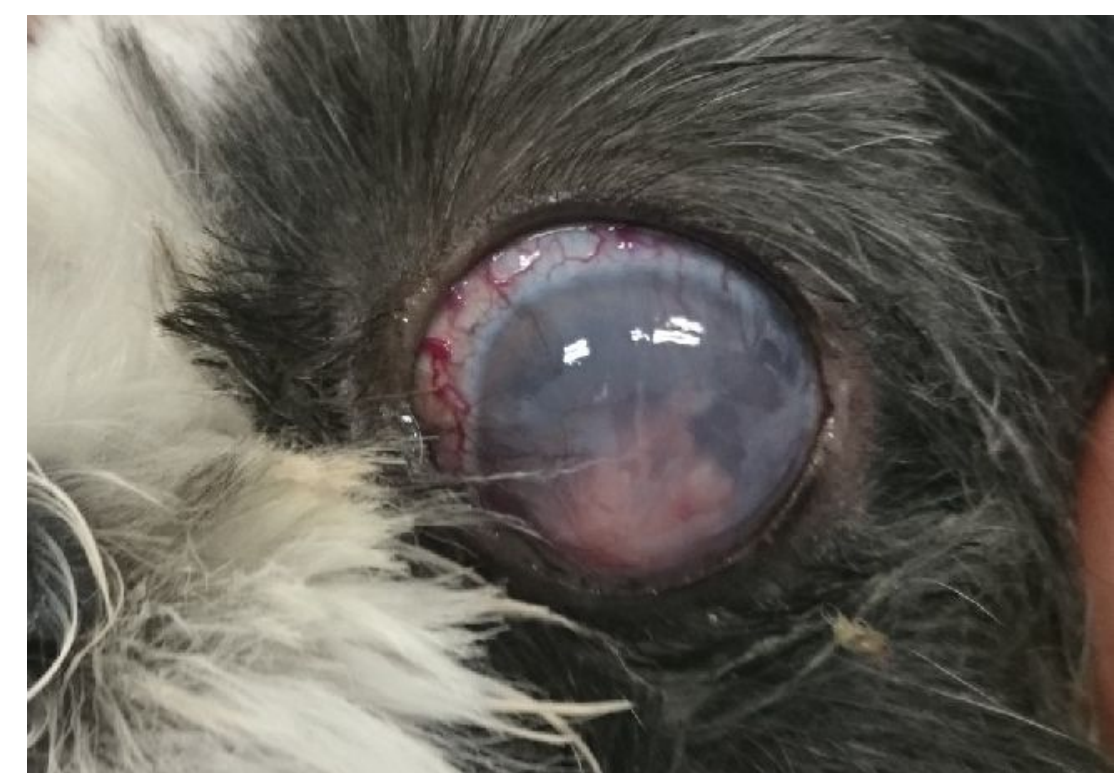

FIGURA 1: Paciente canina, fêmea, da raça Shih tzu, três anos de idade apresentando buftalmia em olho esquerdo, vasos episclerais ingurgitados, buftalmia e edema de córnea.

Fonte: Andréia Amaral (2018).

No dia do procedimento, constatada a higidez aos exames clínicos e laboratoriais (Hemograma, uréia, creatinina, FA, ALT), procedeu-se o protocolo préanestésico, em que realizou-se a aplicação de $0,03 \mathrm{mg} / \mathrm{kg}$ de acepromazina e 0,5 $\mathrm{mg} / \mathrm{kg}$ de morfina, a indução a anestesia geral foi realizada com a dose de $5 \mathrm{mg} / \mathrm{kg}$ de propofol e $0,3 \mathrm{mg} / \mathrm{kg}$ de midazolam, a paciente foi entubada e a manutenção da anestesia foi realizada com isoflurano.

Com a paciente anestesiada, em decúbito lateral direito, foi realizada a antissepsia da superfície ocular utilizando $250 \mathrm{ml}$ de solução fisiológica acrescida de $2,5 \mathrm{ml}$ de solução de iodopovidona. Uma agulha $25 \times 8 \mathrm{~mm}$ foi posicionada na porção superior, cranial do bulbo ocular, e introduzida na câmara vítrea, de onde se retirou 
cerca $0,7 \mathrm{ml}$ de vítreo. Ato contínuo, aplicou-se $0,5 \mathrm{ml}$ de solução de gentamicina a $2 \%$ e $0,2 \mathrm{ml}$ de solução de dexametesona a $4 \%$.

O pós-operatório foi instituído a base de solução de tramadol gotas $(2 \mathrm{mg} / \mathrm{kg} \mathrm{a}$ cada 12 horas, por via oral), dipirona gotas ( $25 \mathrm{mg} / \mathrm{kg}$ a cada 12 horas, por via oral) e meloxicam comprimidos $(0,1 \mathrm{mg} / \mathrm{kg}$ a cada 24 horas, por via oral), por 5 dias, e colírios a base de prednisolona ( 1 gota a cada 8 horas) e a base de diclofenaco (1 gota a cada 8 horas) por 15 dias, além do uso de colar elisabetano. Decorridos 15 dias do procedimento cirúrgico, o paciente apresentou diminuição do bulbo ocular, diminuição da opacidade corneal de forma marcante e PIO de $11 \mathrm{mmHg}$. No entanto, ainda mostrava discreta hiperemia e ingurgitamento dos vasos episclerais, recomendando-se a continuidade do tratamento por mais sete dias. Após sete dias, foi verificada ausência de hiperemia e ingurgitamento dos vasos episclerais (Figura 2), e PIO de $10 \mathrm{mmHg}$, suspendendo-se assim, os colírios e o uso do colar elisabetano.

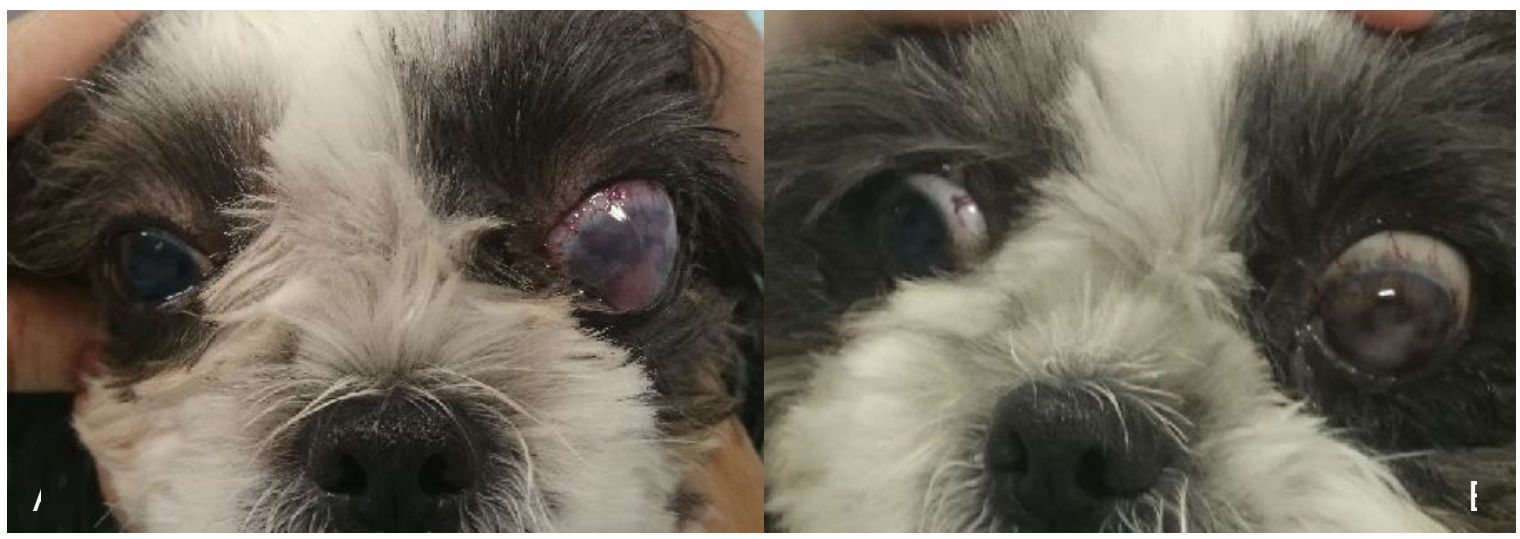

FIGURA 2: (A) Paciente fêmea, da raça Shih Tzu, três anos de idade, antes da injeção intravitrea de gentamicina, evidenciando acentuadas buftalmia e ingurgitamento dos vasos episclerais (B) Paciente 21 dias dias após o procedimento, apresentando diminuição no tamanho do globo ocular, ausência de hiperemia e de ingurgitamento dos vasos episclerais.

Fonte: Andréia Amaral (2018).

\section{RESULTADOS E DISCUSSÃO}

De acordo com os exames oftálmicos e principalmente com a tonometria, foi possível fechar o diagnóstico deste caso em Glaucoma. Devido ao histórico de trauma, o glaucoma foi classificado como secundário, corroborando com a literatura descrita por Oliver (2015), em que este relata que o glaucoma secundário se origina a partir de um evento que possibilite seu desenvolvimento, como nos casos de catarata, traumatismo, uveíte, defeitos zonulares ou neoplasia.

Assim como descrito por Suhett (2014), o histórico de trauma, os sinais clínicos de olho em buftalmia, vasos episclerais ingurgitados, ausência de resposta à ameaça e epífora são condizentes com as alterações encontradas em cães glaucomatosos, e para o diagnóstico definitivo a tonometria ocular torna-se uma avaliação excelente.

$\mathrm{Na}$ tentativa de preservar o olho do paciente, tratamento medicamentoso pode ser prescrito nos casos agudos, mas é importante ressaltar que não são todos os casos que respondem a esta opção (OLIVER, 2015). Análogos de prostaglandinas podem ser utilizados de forma tópica para tratamento de médio a 
longo prazo do glaucoma, e pode ser combinada com outras drogas como inibidores de anidrase carbônica e beta-bloqueadores para promover um efeito aditivo (OLIVER, 2015).

O timolol, medicamento utilizado neste caso, é um beta bloqueador não seletivo, causando inibição dos receptores beta ou da ativação de receptores alfaadrenérgicos no músculo da íris, podendo causar miose em cães (WLIKIE e LATIMER, 1991). Este medicamento na concentração de $0,5 \%$ demonstrou máximo descréscimo na PIO no sétimo dia de tratamento. Este deve ser administrado em intervalos regulares de oito ou 12 horas (MAEHARA et al, 2004), assim como relatado.

A junção de Dorzolamida a $2 \%$ e maleato de timolol a $0,5 \%$, formulação comercial (Cosopt), apresenta efeito aditivo em cães com glaucoma (PLUMER, 2006), porém podem apresentar efeitos adversos como blefarite, que tende a cessar com a descontinuidade do tratamento (WILLIS, 2004). Neste relato de caso, este tratamento não apresentou efeitos adversos assim como não foi eficaz, corroborando as descrições da literatura, em que a maioria dos casos não são responsivos ao tratamento medicamentoso (OLIVER, 2015).

Quando há falha no tratamento medicamentoso empregam-se técnicas cirúrgicas que objetivam diminuir a produção do humor aquoso, por meio de lesões no corpo ciliar ou manobras que incrementam a sua drenagem, aumentando a vazão através de vias alternativas (GELATT e GELATT, 2001). Mas, mesmo que haja sucesso no tratamento cirúrgico, frequentemente haverá necessidade de se utilizar medicações tópicas no pós-cirúrgico (OLIVER, 2015).

A técnica utilizada no presente relato é denominada ablação química do corpo ciliar ou cicloablação (OLIVER, 2015), por gentamicina. Nesta técnica ocorre a destruição dos processos ciliares, que são responsáveis pela produção de humor aquoso (OLIVER, 2015) por meio de injeção de uma droga citotóxica, frequentemente a gentamicina, dentro da câmara vítrea (WELIHOZKIY, 2017). No entanto, o mecanismo pelo qual a gentamicina causa a destruição do epitélio do corpo ciliar ainda não é totalmente esclarecida (RANKIN et al, 2016).

Oliver (2015) descreveu que este tipo de intervenção causa dor acentuada tanto no pós-operatório imediato quanto no prolongado, tendo índice de sucesso baixo e inúmeras complicações, por este motivo tal técnica deve ser utilizada em casos específicos.

O paciente deste relato não apresentou complicações pós-operatórias e respondeu de forma eficaz ao tratamento cirúrgico, teve sua PIO reduzida e as demais alterações oculares normalizadas. Também foram empregados medicamentos analgésicos e antinflamatórios à base de meloxicam, dipirona sódica e tramadol por via oral e prednisolona e diclofenaco tópicos, visando a analgesia multimodal, já que se trata de uma técnica sabidamente dolorosa (OLIVER, 2015).

Suhett (2014) descreveu em seu relato a utilização de $0,4 \mathrm{ml}$ de sulfato de gentamicina isolada, sendo aplicada no interior da câmera vítrea do globo ocular. Neste relato foram retirados $0,7 \mathrm{ml}$ de humor vítreo e aplicados $0,5 \mathrm{ml}$ de solução de gentamicina a $2 \%+0,2 \mathrm{ml}$ de solução de dexametasona a $4 \%$. Ambas as técnicas apresentaram eficácia na correção do glaucoma.

A remoção do globo ocular de forma cirúrgica é recomendada em casos de glaucoma secundário a neoplasias intraoculares, ou em inflamações crônicas e condições traumáticas associadas à cegueira (MAGGIO, 2015), também nos casos de panoftalmia, perfuração do globo ocular, trauma severo com hemorragia 
intraocular ou quando o tutor não consegue fazer o tratamento prolongado (WELIHOZKIY, 2017). Porém não há uma boa aceitação pelos tutores, que preferem, caso seja possível, manter o globo ocular por estética.

Em contraste com a enucleação, a técnica da ablação do corpo ciliar por droga citotóxica é indicada somente para tratar e promover conforto no estágio final da cegueira, nos casos de olhos glaucomatosos que não responderam a tratamento medicamentoso (WELIHOZKIY, 2017). Esta técnica não é recomendada para felinos, devido ao risco de desenvolvimento de sarcoma intraocular, porém é pode ser empregada em cães sem este risco (WELIHOZKIY, 2017). A complicação mais comum no pós-operatório imediato desta técnica é hemorragia intraocular (WELIHOZKIY, 2017), o que não ocorreu no paciente deste relato.

\section{CONCLUSÃO}

A técnica de ablação do corpo ciliar por gentamicina mostrou-se eficaz em reduzir a PIO, e consequentemente normalizar as alterações oculares, sendo principalmente indicada em alguns casos de glaucoma secundário em que já há buftalmia, dor e cegueira. Além disso, com esse tipo de tratamento é possível a manutenção do globo ocular do paciente, preservando a estética e sendo bem aceita pelos tutores, além de ser menos invasiva que a evisceração e a enucleação do globo ocular.

\section{REFERÊNCIAS}

BRAS, D.; MAGGIO, F. Surgical Treatment of Canine Glaucoma Cyclodestructive Techniques. Veterinary Clinics of North America: Small Animal Practice, v. 45, p. 1283-1305, 2015. Disponível em <http://dx.doi.org/10.1016/j.cvsm.2015.06.007>. doi: 10.1016/j.cvsm.2015.06.007.

BROADWATER, J. J.; DVM; Jamie J. Schorling, DVM, MS; lan P. Herring, DVM, MS; François Elvinger. Effect of body position on intraocular pressure in dogs without glaucoma. American Journal of Veterinary Research, v. 69, n. 4, p 527530, 2008. Disponível em <https://doi.org/10.2460/ajvr.69.4.527>. doi: 10.2460/ajvr.69.4.527.

BROOKS, D. E. Glaucoma. In.: HERRERA, D. Oftalmologia Clínica em Animais de Companhia. São Paulo: MedVet, 2008, p. 195-203.

CAPRIOLI, J.; COLEMAN, A. L. Blood pressure, perfusion pressure, and glaucoma. American Journal of Ophthalmology, v. 149, n. 5, p. 704-712, 2010. Disponível em <https://doi.org/10.1016/j.ajo.2010.01.018>. doi: 10.1016/j.ajo.2010.01.018

EURIDES, D.; SILVA, L. A. F. Manual de Cirurgia Oftálmica Veterinária. Curitiba: Medvep, 2013, 204 p.

GELATT, K. N.; GELATT, J. P. Surgery of nasolacrimal apparatus and tear systems. In: Small animal ophthalmic surgery: practical techniques for the veterinarian. Oxford: Butterworth \& Heinemann. p.125-141. 2001. 
KATO, K; VAN DER WOERDT, A. Effect of Long-term Topical Application of $0.005 \%$ Latanoprost on Intraocular Pressure Uncontrolled by Multiple or Single Drug Therapy in Dogs with Secondary Glaucoma. Journal of Veterinary Science \& Medical Diagnosis, v. 6, n. 1, 2017. Disponível em: <https://doi.org/10.4172/2325-9590>. doi: 10.4172/2325-9590.

MAEHARA, S. et al. Effects of topical nipradilol and timolol on intraocular pressure, facility outflow, arterial blood pressure and pulse rate in dogs. Veterinary Ophthalmology, Oxford, v. 7,n. 3, p.147-50, 2004. Disponível em: <https://doi.org/10.1111/j.1463-5224.2004.04010.x>. doi: 10.1111/j.14635224.2004.04010.x.

MAGGIO, F. Surgical Treatment of Canine Glaucoma: Filtering and End Stage Glaucoma Procedures. Veterinary Clinics of North America: Small Animal Practice, n. 45, p.1261-1282. 2015. Disponível em:<http://dx.doi.org/10.1016/j.cvsm.2015.06.008> doi: 10.1016/j.cvsm.2015.06.008.

MARTINS, B. C.; RIBEIRO, A. P.; LAUS, J. L.; ORTIZ, J. P. D. Glaucoma. In: LAUS, J. L. Oftalmologia Clínica e Cirúrgica em Cães e Gatos. São Paulo: Rocca, 2009. p. 151-167

MILLER, P. E; BENTLEY, E. Clinical Signs and Diagnosis of the Canine Primary Glaucomas. Veterinary Clinics of North America: Small Animal Practice, n. 45, p. 1183-1212, 2015. Disponível em < http://dx.doi.org/10.1016/j.cvsm.2015.06.006>. doi: 10.1016/j.cvsm.2015.06.006,

OFRI, R.; NARFSTROM, K. Light at the end of the tunnel? Advances in the understanding and treatment of glaucoma and inherited retinal degeneration. The Veterinary Journal. v. 174, n. 1, p. 10-22, 2007. Disponível em: <https://doi.org/10.1016/j.tvjl.2006.08.014>. doi: 10.1016/j.tvjl.2006.08.014

OLIVER, J. Canine Glaucoma. Companion animal, v. 20, n. 2, 2015. Disponível em: <https://doi.org/10.12968/coan.2015.20.2.114>. doi: 10.12968/coan.2015.20.2.114.

ORIÁ, A. P.; PEREIRA, P. M.; LAUS, J. L. Uveitis in dogs infected with Ehrlichia canis. Ciência Rural, v. 34, n. 4, p. 1289-1295, 2004. Disponível em <http://dx.doi.org/10.1590/S0103-84782004000400055>. doi: 10.1590/S010384782004000400055 .

PIZZIRANI, S. Definition, Classification, and Pathophysiology of Canine Glaucoma. Veterinary Clinics of North America: Small Animal Practice, v. 45, n. $6, \quad$ p. 1127-1157, 2015. Disponível em <http://dx.doi.org/10.1016/j.cvsm.2015.06.002>. doi: 10.1016/j.cvsm.2015.06.002. 
PIZZIRANI, S.; GONG, H. Functional Anatomy of the Outflow Facilities. Veterinary Clinics of North America: Small Animal Practice, v, 45, p. 11011126, 2015. Disponível em <http://dx.doi.org/10.1016/j.cvsm.2015.06.005>. doi: 10.1016/j.cvsm.2015.06.005.

PLUMER, C. E, MACKAY, E, O; GELATT, K, N. Comparison of the effects of topical administration of a fixed combination of dorzolamide-timol to monotherapy with timolol or dorzolamide on IOP heart rate in glaucomatous beagles. Veterinary Ophthalmology, v. 9, n. 4, p. 245-249, 2006. Disponível em: <https://doi.org/10.1111/j.1463-5224.2006.00469.x>. doi: 10.1111/j.14635224.2006.00469.x.

PLUMMER, C. E; REGNIER, A; GELATT, K. N. The canine glaucomas. In: GELATT, K. N; GILGER, B. C; KERN, T. J. Veterinary ophthalmology. 5ed. Hoboken (NJ): John Wiley \& Sons, p. 1050-145, 2013.

PUMPHREY, S. Canine Secundary Glaucomas. Veterinary Clinics of North America: Small Animal Practice, v. 45, p. 1335-1364, 2015. Disponível em: <http://dx.doi.org/10.1016/j.cvsm.2015.06.009>. doi: 10.1016/j.cvsm.2015.06.009.

RANKIN, A. J._Measurement of plasma gentamicin concentrations postchemical ciliary body ablation in dogs with chronic glaucoma. Veterinary Ophthalmology, v. 19, n. 1, p. 57-62. 2016. Disponível em: <https://www.ncbi.nlm.nih.gov/pubmed/25688853> DOI:10.1111/vop.12258

SANDALON, S.; KÖNNECKE, B.; LEVKOVITCH-VERBIN, H.; SIMONS, M.; HEIN, K.; SÄTTLER, M.B.; BÄHR, M.; OFRI, R. Functional and structural Evaluation of lamotrigine treatment in rat models of acute and chronic ocular hypertension. Experimental Eye Research, v. 115, p. 47-56, 2013. Disponível em <https://doi.org/10.1016/j.exer.2013.06.018>. doi: 10.1016/j.exer.2013.06.018.

SEBBAG, L; ALLBAUGH, R. A.; STRAUSS, R. A.; STRONG, T. D.; WEHRMAN, R. F., et al. MicroPulse ${ }^{\mathrm{TM}}$ transscleral cyclophotocoagulation in the treatment of canine glaucoma: Preliminary results (12 dogs). Veterinary Ophthalmology, p. 1-8, 2018. Disponível em <http://dx.doi.org/10.1111/vop.12603. doi: 10.1111/vop.12603.

SLATTER, D. Fundamentos de Oftalmologia Veterinária. 3.ed.; São Paulo: Roca, 2005. 712 p.

STROM, A. R.; HÄSSIG, M.; IBURG, T. M.; SPIESS, B. M. Epidemiology of canine glaucoma presented to University of Zurich from 1995 to 2009. Part 1: congenital and primary glaucoma (4 and 123 cases). Veterinary Ophthalmology, v. 14, n. 2, p. 121- 126, 2011. Disponível em: < https://doi.org/10.1111/j.1463-5224.2010.00855.x>. doi: 10.1111/j.14635224.2010.00855.x 
SUHETT, W. G. Avaliação clínica da associação da técnica de ablação farmacológica e flap de terceira pálpebra como tratamento em caso de glaucoma canino com úlcera de córnea como agravante - relato de caso. Revista de Ciência Veterinária e Saúde Pública, v. 1, n. 2, p. 135-140, 2014. Disponível em: <http://periodicos.uem.br/ojs/index.php/RevCiVet/article/view/19908/pdf_47)>

TURNER, S. M. Oftalmologia em pequenos animais. Rio de Janeiro: Elsevier, 2010. $384 \mathrm{p}$.

VEIGA, C. C. P.; BONFIM, P. C.; OLIVEIRA, P. C. Aspecto ultrassonográfico da uveíte unilateral canina - relato de caso. Revista Brasileira de Medicina Veterinária, v. $35, \quad$ n. 1 ; $p .11-14,2013$. Disponível em < rbmv.org/index.php/BJVM/article/download/577/440/>.

WELIHOZKIY, A. Enucleation \& Pharmacologic Ciliary Body Ablation of the Eye. BluePearl Veterinary Partners Sarasota, Florida. 2017. Disponível em: $<$ https://www.cliniciansbrief.com/ >

WERNE, A.; HARRIS, A.; MOORE, D.; BENZION, I.; SIESKY, B. The circadian variations in systemic blood pressure, ocular perfusion pressure, and ocular blood flow: Risk factors for glaucoma? Survey of Ophthalmology. v. 53, n. 6, p. 559567, 2008. Disponível em <https://doi.org/10.1016/j.survophthal.2008.08.021>. doi: 10.1016/j.survophthal.2008.08.021.

WILKIE, D. A.; LATIMER, C. A. Effects of topical administration of timolol maleate on intraocular pressure and pupil size in dogs. American Journal of Veterinary Research, Schaumburg, v. 52, n. 3, p. 432-435,n. 1991. Disponível em <https://europepmc.org/abstract/med/2035917>.

WILLIS, A.M. Ocular hypotensive drugs. Veterinary Clinics of North America: Small Animal Practice, Philadelphia, v. 34, n. 3, p. 755-76, 2004. 